\title{
Nondestructive identification and accurate isolation of single cells through a chip with Raman optical tweezers
}

Teng Fang ${ }^{\dagger}$, Wenhao Shang ${ }^{\ddagger}$, Chang Liu ${ }^{\dagger}$, Jingjing Xu ${ }^{\S}$, Dongping Zhao ${ }^{\dagger}, \quad$ Yaoyao Liu and Anpei $\mathrm{Ye}^{*,+,+}$

${ }^{\dagger}$ Key Laboratory for the Physics and Chemistry of Nanodevices, Department of Electronics, School of Electronics Engineering and Computer Science, Peking University, Beijing 100871, China.

${ }^{\ddagger}$ Biomed-X Research Center, Academy for Advanced Interdisciplinary Studies, Peking University, Beijing 100871, China.

${ }^{\S}$ Peking-Tsinghua Center for Life Sciences, Peking University, Beijing 100871, China.

*E-mail: yap@pku.edu.cn

\section{Contents}

Table S1. Assignments of observed Raman bands in the spectra for different BGC823 cancer cells (Cell A-C)

Table S2. Assignments of observed Raman bands in the spectrum for Cell D (a leukocyte)

Table S3. Assignments of observed Raman bands in the spectrum for Cell E (an erythrocyte) S-3

Figure S1. Raman spectrum of an E. coli cell (Cell F) S-3

Table S4. Assignments of observed Raman bands in the spectrum for Cell F (an E. coli cell)

Figure S2. Graphic cell distribution in the gourd-like reservoir at the moment before singlecell extraction. S-4 
Table S1. Assignments of observed Raman bands in the spectra for different BGC823 cancer cells (Cell A-C).

\begin{tabular}{|c|c|c|c|c|c|}
\hline \multicolumn{2}{|c|}{ Raman band $\left(\mathrm{cm}^{-1}\right)$} & \multirow[t]{2}{*}{ Assignment } & \multicolumn{2}{|c|}{ Raman band $\left(\mathrm{cm}^{-1}\right)$} & \multirow[t]{2}{*}{ Assignment } \\
\hline Previous works & $\begin{array}{l}\text { Present } \\
\text { work }\end{array}$ & & Previous works & $\begin{array}{l}\text { Present } \\
\text { work }\end{array}$ & \\
\hline $688^{1}$ & 686 & Amide IV & $1206^{2}$ & 1205 & Phe, Amide III; $\delta(=\mathrm{CH})$ in lipids \\
\hline$?$ & 740 & $\begin{array}{l}\text { possible both contributions from bands at } 732 \mathrm{~cm}^{-1}( \\
\text { A, Amide IV })^{1} \text { and } 745 \mathrm{~cm}^{-1}(\mathrm{~A}, \mathrm{~T}, \mathrm{C}, \operatorname{Trp}(\text { sym br), } \\
\text { Cytochrome C })^{2,3}\end{array}$ & $\begin{array}{l}1240^{4,5} \\
1245^{6,7}\end{array}$ & 1240 & A, T; Amide III \\
\hline $785^{2}, 791^{1}$ & 790 & $\mathrm{C}, \mathrm{T}, \mathrm{U}$ & $1310^{2}$ & 1312 & $\begin{array}{l}\text { G; Amide III, Cytochrome } \mathrm{C} ; \gamma\left(\mathrm{CH}_{2}\right) \text { and } v(\mathrm{CN}) \text { in } \\
\text { lipids }\end{array}$ \\
\hline $841^{3}$ & 841 & Ribose, Ribose-phosphate & $1337^{2}$ & 1335 & A, G; collagen, Cytochrome $\mathrm{C}, \delta(\mathrm{CH})$ in proteins; \\
\hline $859^{1}$ & 860 & Tyr, tyr Fermi doublet & $1446^{7}, 1447^{2}$ & 1447 & $\begin{array}{l}\gamma\left(\mathrm{CH}_{3} \mathrm{CH}_{2}\right) \text { in lipids } \\
\delta(\mathrm{CH}) \text { in nucleic acids, } \delta\left(\mathrm{CH}_{2}\right) \text { in proteins, } \delta\left(\mathrm{CH}_{3}\right) \\
\text { in lipids }\end{array}$ \\
\hline $938^{5}, 944^{1}$ & 940 & $v(\mathrm{C}-\mathrm{C} \mathrm{bk})$ in proteins & $1473^{6}$ & 1473 & G \\
\hline $1001^{7}, 1004^{2}$ & 1004 & Phe (phenyl ring) & $1577^{2}$ & 1575 & A, G; Phe, Cytocrome C \\
\hline $1031^{6}, 1032^{5}$ & 1030 & Phe, $v(\mathrm{C}-\mathrm{N})$ in proteins & $1607^{2}$ & 1607 & Phe, Tyr; $v(\mathrm{C}=\mathrm{C})$ in lipids \\
\hline $1090^{2}$ & 1093 & $\mathrm{PO}_{2}$ bk in nucleic acids, $\mathrm{Phe}, v(\mathrm{C}-\mathrm{N})$ in proteins & $1660^{5}, 1664^{1}$ & 1660 & Amide $\mathrm{I}, v(\mathrm{C}=\mathrm{C})$ in lipids \\
\hline $1120^{2}$ & 1120 & $\begin{array}{l}\mathrm{PO}_{2} \text { bk in nucleic acids, } v(\mathrm{C}-\mathrm{N}) \text { in proteins, } \\
\text { Cytochrome C }\end{array}$ & & & \\
\hline
\end{tabular}

Abbreviations: $v$, stretching modes; $\delta$, in-plane bending modes; $\gamma$, out-of-plane bending modes; br, breathing; A, adenine; G, guanine; C, cytosine; T, thymine; U, uracil; Tyr, tyrosine; Trp, tryptophan; Phe, phenylalanine; pyr, pryrole in the porphyrin form; sym, symmetric; asym, asymmetric; bk, backbone.

Table S2. Assignments of observed Raman bands in the spectrum for Cell D (a leukocyte).

\begin{tabular}{|c|c|c|c|c|c|}
\hline \multicolumn{2}{|c|}{ Raman band $\left(\mathrm{cm}^{-1}\right)$} & \multirow[t]{2}{*}{ Assignment } & \multicolumn{2}{|c|}{ Raman band $\left(\mathrm{cm}^{-1}\right)$} & \multirow[t]{2}{*}{ Assignment } \\
\hline Previous works & $\begin{array}{l}\text { Present } \\
\text { work }\end{array}$ & & Previous works & $\begin{array}{l}\text { Present } \\
\text { work }\end{array}$ & \\
\hline $688^{1}$ & 687 & Amide IV & $1232^{3,4}$ & 1232 & C, U, Amide III \\
\hline $\begin{array}{l}745^{2}, 754^{5} \\
755^{1}\end{array}$ & 750 & $\begin{array}{l}\text { A,T,C; Trp, Cytochrome C, some small contributions } \\
\text { from Phe and Try }\end{array}$ & $\begin{array}{l}1240^{4,5} \\
1245^{6,7}\end{array}$ & 1246 & Amide III (bonds $\beta$ sheets) \\
\hline $785^{2}, 791^{1}$ & 790 & $\mathrm{C}, \mathrm{T}, \mathrm{U}$ & $\begin{array}{l}1300^{3} \\
1301^{5}, 1310^{2}\end{array}$ & 1305 & $\begin{array}{l}\text { A,G,C; Amide III, Cytochrome C; } \\
\delta\left(\mathrm{CH}_{2}\right) \text { and } v(\mathrm{C}-\mathrm{N}) \text { in lipids }\end{array}$ \\
\hline $859^{1}$ & 858 & Tyr, Tyr Fermi doublet & $1337^{2}$ & 1335 & A, G; collagen, Cytochrome C, $\delta(\mathrm{CH})$ in proteins; \\
\hline $902^{1}$ & 903 & Nucleic acid bk; possible $\mathrm{CH}_{2}$ rocking from lipids & $1370^{2}$ & 1367 & $\begin{array}{l}\gamma\left(\mathrm{CH}_{3} \mathrm{CH}_{2}\right) \text { in lipids } \\
\mathrm{A}, \mathrm{T}, \mathrm{G} \text { in nucleic acids; Cytochrome C }\end{array}$ \\
\hline $929^{2}$ & 929 & DNA bk; $v(\mathrm{C}-\mathrm{C})$ in proteins & $1446^{7}, 1447^{2}$ & 1445 & $\begin{array}{l}\delta(\mathrm{CH}) \text { in nucleic acids, } \delta\left(\mathrm{CH}_{2}\right) \text { in proteins, } \delta\left(\mathrm{CH}_{3}\right) \\
\text { in lipids }\end{array}$ \\
\hline $1001^{7}, 1004^{2}$ & 1003 & Phe (phenyl ring) & $1480^{8}, 1485^{2}$ & 1479 & $\mathrm{~A}, \mathrm{G}, \mathrm{DNA} ; \delta(\mathrm{CH})$ in proteins \\
\hline $1049^{9}$ & 1049 & $\mathrm{C}-\mathrm{O}$ and $\mathrm{C}-\mathrm{N}$ in proteins & $1507^{3}$ & 1505 & A \\
\hline $1090^{2}$ & 1090 & $\mathrm{PO}_{2}$ bk in nucleic acid, Phe, $v(\mathrm{C}-\mathrm{N})$ in proteins & $1577^{2}$ & 1573 & A, G; Phe, Cytocrome C \\
\hline $1120^{2}$ & 1120 & $\begin{array}{l}\mathrm{PO}_{2} \text { bk in nucleic acid, } v(\mathrm{C}-\mathrm{N}) \text { in proteins, } \\
\text { Cytochrome C }\end{array}$ & $1617^{2}$ & 1617 & Tyr, Trp; $C=C$ in lipids \\
\hline $1200^{5}$ & 1198 & A, T, Tyr, Phe, Amide III & $1654^{9}, 1656^{6}$ & 1652 & Amide $\mathrm{I}, a$-helix in proteins ; $v(\mathrm{C}=\mathrm{C})$ in lipids \\
\hline
\end{tabular}


Table S3. Assignments of observed Raman bands in the spectrum for Cell E (an erythrocyte).

\begin{tabular}{|c|c|c|c|c|c|}
\hline \multicolumn{2}{|c|}{ Raman band $\left(\mathrm{cm}^{-1}\right)$} & \multirow[t]{2}{*}{ Assignment } & \multicolumn{2}{|c|}{ Raman band $\left(\mathrm{cm}^{-1}\right)$} & \multirow[t]{2}{*}{ Assignment } \\
\hline Previous works & $\begin{array}{l}\text { Present } \\
\text { work }\end{array}$ & & Previous works & $\begin{array}{l}\text { Present } \\
\text { work }\end{array}$ & \\
\hline $688^{1}$ & 686 & Amide IV & $1301^{5}, 1305^{10}$ & 1299 & $\delta\left(\mathrm{C}_{\mathrm{m}} \mathrm{H}\right)$ \\
\hline$?$ & 765 & $\begin{array}{l}\text { possible contributions from bands at } 754 \mathrm{~cm}^{-1}(\mathrm{pyr} b r \text {, } \\
\text { Cytochrome C) })^{1,10,11} \text { and } 789 \mathrm{~cm}^{-1}(\mathrm{pyr} \text { br })^{10}\end{array}$ & $1336^{11}$ & 1335 & $v(\text { pyr quarter ring })_{\text {sym }}$ \\
\hline $974^{10}$ & 975 & $\gamma\left(\mathrm{C}_{\mathrm{a}} \mathrm{H}=\right)$ & $1356^{10}, 1358^{11}$ & 1354 & $v(\text { pyr half ring })_{\text {sym }}$ \\
\hline $992^{10}$ & 991 & $v\left(\mathrm{C}_{\beta} \mathrm{C}_{1}\right)_{\text {asym }}$ & $1384^{10}$ & 1385 & $v(\text { pyr half ring })_{\text {sym }}$ \\
\hline $1003^{10}$ & 1003 & Phe (phenyl ring) & $1423^{5}$ & 1419 & $v\left(\mathrm{C}_{a} \mathrm{C}_{\mathrm{m}}\right)_{\mathrm{sym}}$ \\
\hline $1072^{10}$ & 1072 & $\delta\left(=\mathrm{C}_{\mathrm{b}} \mathrm{H}_{2}\right)_{4}$ & $1454^{1}, 1460^{12}$ & 1460 & $v\left(\mathrm{CH}_{2} \mathrm{CH}_{3}\right)$ in protein \\
\hline $1085^{10}$ & 1084 & $\delta\left(=\mathrm{C}_{\mathrm{b}} \mathrm{H}_{2}\right)_{4}$ & $1547^{10}$ & 1544 & $v\left(\mathrm{C}_{\beta} \mathrm{C}_{\beta}\right)$ \\
\hline $1120^{2}$ & 1117 & $v(\mathrm{C}-\mathrm{N})$ in proteins, Cytochrome C & $1582^{10}$ & 1579 & $v\left(\mathrm{C}_{\alpha} \mathrm{C}_{\mathrm{m}}\right)_{\text {asym }}$ \\
\hline $1170^{10}$ & 1168 & $v(\text { pyr half-ring })_{\text {asym }}$ & $1604^{11}$ & 1601 & $v(\mathrm{C}=\mathrm{C})_{\text {vinyl }} / v\left(\mathrm{C}_{\mathrm{a}}=\mathrm{C}_{\mathrm{b}}\right)$ \\
\hline $1206^{2}$ & 1205 & Phe, Amide III & & & \\
\hline
\end{tabular}

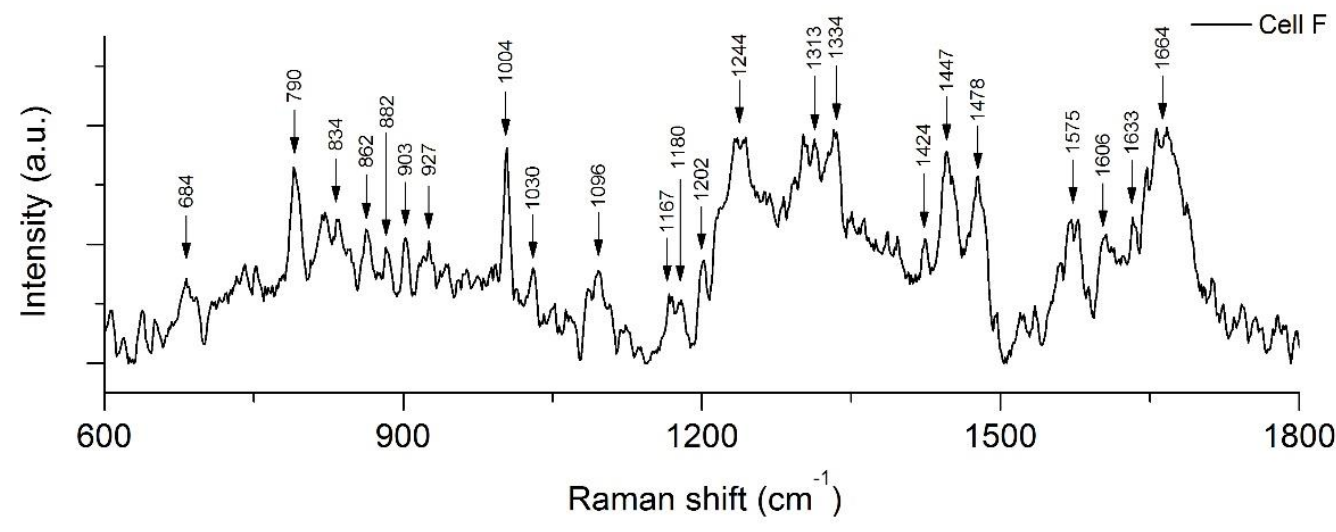

Table S4. Assignments of observed Raman bands in the spectrum for Cell F (an E. colicell).

\begin{tabular}{|c|c|c|c|c|c|}
\hline \multicolumn{2}{|c|}{ Raman band $\left(\mathrm{cm}^{-1}\right)$} & \multirow[t]{2}{*}{ Assignment } & \multicolumn{2}{|c|}{ Raman band $\left(\mathrm{cm}^{-1}\right)$} & \multirow[t]{2}{*}{ Assignment } \\
\hline Previous works & $\begin{array}{l}\text { Present } \\
\text { work }\end{array}$ & & Previous works & $\begin{array}{l}\text { Present } \\
\text { work }\end{array}$ & \\
\hline $685^{5}$ & 684 & G & $1206^{2}$ & 1202 & Phe, Amide III; $\delta(=\mathrm{CH})$ in lipids \\
\hline $783^{13}, 791^{1}$ & 790 & $\mathrm{C}, \mathrm{T}, \mathrm{U}$ & $1249^{5,14}$ & 1244 & A, T; Amide III \\
\hline $830^{14}, 833^{2}$ & 834 & $\begin{array}{l}v\left(\mathrm{PO}_{2}\right)_{\text {asym }} \text { in nucleic acids; Tyr; } \\
v(\text { diester })_{\text {sym }} \text { in lipids }\end{array}$ & $1308^{13}, 1309^{1}$ & 1313 & Amide III; A, C \\
\hline $857^{13}, 859^{1}$ & 862 & $\mathrm{~T}, \mathrm{Tyr}$ & $1337^{2}$ & 1334 & A, G; collagen, Cytochrome $\mathrm{C}, \delta(\mathrm{CH})$ in proteins; \\
\hline $884^{1}$ & 882 & nucleic acid bk & $1420^{2}$ & 1424 & $\begin{array}{l}\gamma\left(\mathrm{CH}_{3} \mathrm{CH}_{2}\right) \text { in lipids } \\
\delta(\mathrm{CH}) \text { in nucleic acids; } \delta\left(\mathrm{CH}_{2}\right) \text { in proteins; } \\
\delta\left(\mathrm{CH}_{3}\right) \text { in lipids }\end{array}$ \\
\hline $899^{13}$ & 903 & DNA bk & $\begin{array}{l}1447,1450^{14} \\
1453^{13}\end{array}$ & 1447 & $\begin{array}{l}\delta(\mathrm{CH}) \text { in nucleic acids; } \delta\left(\mathrm{CH}_{2}\right) \text { in proteins; } \\
\delta\left(\mathrm{CH}_{3}\right) \text { in lipids }\end{array}$ \\
\hline $929^{2}$ & 927 & DNA bk; $v(\mathrm{C}-\mathrm{C})$ in proteins & $1485^{3,15}$ & 1478 & A, G \\
\hline $1004^{13,16}$ & 1004 & Phe & $1573^{14}, 1577^{2}$ & 1575 & A, G; Phe, Cytocrome C $(\mathrm{C}=\mathrm{C})$ \\
\hline $1030^{14}, 1031^{6}$ & 1030 & Phe, $v(\mathrm{C}-\mathrm{N})$ in proteins & $1606^{4}$ & 1606 & Phe, Tyr \\
\hline $1095^{13,16}$ & 1096 & DNA: O-P-O- & $?$ & 1633 & $\begin{array}{l}\text { possible contributions from Amide I (1640-1700 } \\
\left.\mathrm{cm}^{-1}\right) \text { and } \operatorname{Trp}\left(1619-1621 \mathrm{~cm}^{-1}\right)^{15}\end{array}$ \\
\hline $1162^{3}, 1163^{1}$ & 1167 & Ribose and weak nucleic acid base vibrations & $1660^{13,14}$ & 1664 & Amide I \\
\hline $1181^{3}$ & 1180 & $\mathrm{G}($ external $v(\mathrm{C}-\mathrm{N}))$ & & & \\
\hline
\end{tabular}




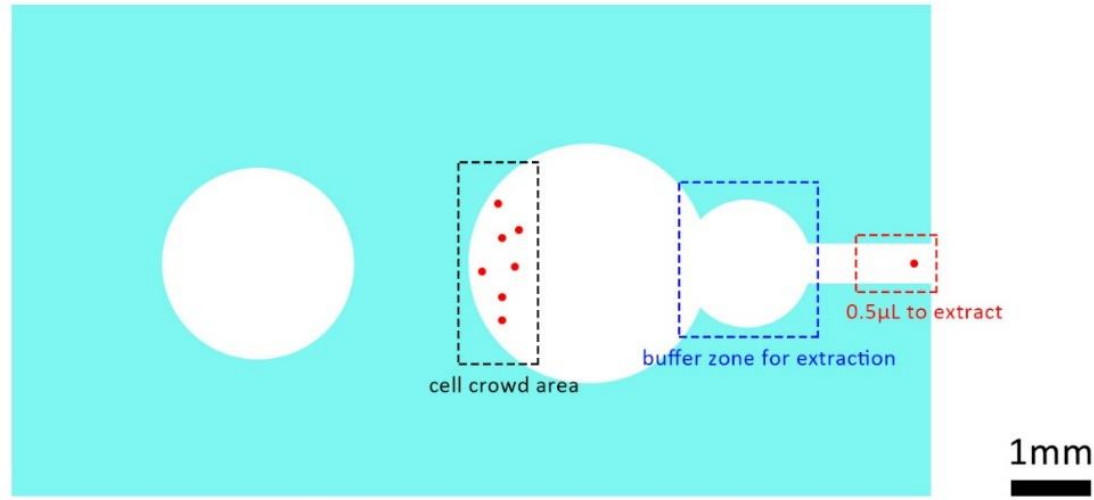

Figure S2. Graphic cell distribution in the gourd-like reservoir at the moment before single-cell extraction.

\section{References}

(1) Hobro, A. J.; Kumagai, Y.; Akira, S.; Smith, N. I. Analyst 2016, 141, 3756-3764.

(2) Manago, S.; Mirabelli, P.; Napolitano, M.; Zito, G.; De Luca, A. C. J Biophotonics 2018, 11, e201700265.

(3) Hobro, A. J.; Standley, D. M.; Ahmad, S.; Smith, N. I. Phys Chem Chem Phys 2013, 15, 13199-13208.

(4) Puppels, G. J.; Garritsen, H. S.; Segers-Nolten, G. M.; Mul, F. F. D.; Greve, J. Biophys J 1991, 60, $1046-1056$.

(5) Bankapur, A.; Zachariah, E.; Chidangil, S.; Valiathan, M.; Mathur, D. PLoS One 2010, 5, e10427.

(6) Jung, G. B.; Lee, Y.J.; Lee, G.; Park, H. K. Biomed Opt Express 2013, 4, 2673-2682.

(7) Zhang, Y.; Jin, L.; Xu, J.; Yu, Y.; Shen, L.; Gao, J.; Ye, A. Analyst 2017, 143, 164-174.

(8) Yazdi, Y.; Ramanujam, N.; Lotan, R.; Mitchell, M. F.; Hittelman, W.; Richardskortum, R. Appl Spectrosc 1999, 53, 82-85.

(9) Austin, L. A.; Osseiran, S.; Evans, C. L. Analyst 2016, 141, 476-503.

(10) Wood, B. R.; Caspers, P.; Puppels, G. J.; Pandiancherri, S.; McNaughton, D. Anal Bioanal Chem 2007, $387,1691-1703$.

(11) Ahlawat, S.; Kumar, N.; Uppal, A.; Kumar Gupta, P.J Biophotonics 2017, 10, 41 5-422.

(12) Krafft, C.; Knetschke, T.; Funk, R. H. W.; Salzer, R. Vib Spectrosc 2005, 38, 85-93.

(13) Neuman, K. C.; Block, S. M. Rev Sci Instrum 2004, 75, 2787-2809.

(14) Schuster, K. C.; Urlaub, E.; Gapes, J. R. Journal of Microbiological Methods 2000, 42, 29-38.

(15) Kruglik, S. G.; Royo, F.; Guigner, J. M.; Palomo, L.; Seksek, O.; Turpin, P. Y.; Tatischeff, I.; Falcon-Perez, J. M. Nanoscale 2019, 11, 1661-1679.

(16) Chan, J. W.; Winhold, H.; Corzett, M. H.; Ulloa, J. M.; Cosman, M.; Balhorn, R.; Huser, T. Cytometry A 2007, 71, $468-474$. 